\title{
UNA SOLA MÉTRICA NO CUENTA TODA LA HISTORIA DE LA PRODUCCIÓN CIENTÍFICA. II. PUBLICACIÓN
}

\section{One metric does not tell the whole story of scientific production. II. Publication}

\author{
Nieves GONZÁLEZ-FERNÁNDEZ-VILLAVICENCIO \\ Universidad de Sevilla. Directora de la Biblioteca de la Facultad de Ciencias Económicas y Empresariales. Sevilla. \\ España. \\ Correspondencia: nievesglez@gmail.com
}

Fecha de recepción: 30 de abril de 2016

Fecha de aceptación: 22 de mayo de 2016

Fecha de Publicación: 10 de junio de 2016

Conflicto de intereses: Los autores declaran no tener conflictos de intereses Imágenes: Los autores declaran haber obtenido las imágenes con el permiso de los pacientes Política de derechos y autoarchivo: se permite el autoarchivo de la versión post-print (SHERPA/RoMEO) Licencia CC BY-NC-ND. Licencia Creative Commons Atribución-NoComercial-SinDerivar 4.0 Internacional ๔ U Universidad de Salamanca. Su comercialización está sujeta al permiso del editor
RESUMEN Introducción: Existen diferentes estrategias de publicación científica que pueden ser aborda- das desde la perspectiva de la propia publicación, calidad de las publicaciones y métricas para su evaluación. Método: Revisión narrativa. Resultados: Descripción de los métodos existentes para detectar los indicios de calidad de las publicaciones y las métricas a distintos niveles que han generado fuentes tradicionales y alternativas, a nivel de contribución indivi- dual, publicación, autor e instituciones. Se consideran tanto métricas tradicionales como las denominadas altmétricas. Conclusiones: Ante la variedad de métricas que ofrecen los pro- veedores de recursos electrónicos, los investigadores deben conocer su existencia y caracte- rísticas, la forma de obtenerlos e interpretarlos para demostrar la calidad de sus publicacio- nes ante terceros.

PALABRAS CLAVE

SUMMARY gestión de la información; publicación científica; visibilidad web; impacto científico; métrica; calidad 


\section{INTRODUCCIÓN}

ESTRATEGIAS DE PUBLICACIÓN. En el primer artículo de esta serie de tres, se presentaron las estrategias de publicación desde la perspectiva de la visibilidad, describiendo entre otros los perfiles del autor. Este segundo artículo enfoca este tema desde la perspectiva de la publicación científica, los indicios de calidad de las publicaciones y sus métricas. Veremos en primer lugar dónde interesa publicar según los indicios de calidad de las publicaciones, las formas de medir y los indicadores del impacto científico.

INDICIOS DE CALIDAD DE LAS PUBLICACIONES: SELECCIÓN DE REVISTAS Y VALORACIÓN DE PUBLICACIONES

Una vez que el investigador ha redactado su artículo la siguiente pregunta es dónde publicarlo, por lo que habrá que realizar una preselección de la revista siguiendo distintas estrategias.

Según la temática del artículo, se realizará una búsqueda con las palabras clave (keywords) del artículo en las bases de datos multidisciplinares de mayor prestigio (Web of Science -WoS- y Scopus) y las especializadas en la materia.

A la hora de seleccionar la revista se tiene en cuenta la calidad de la misma y sobre todo que tenga factor de impacto (FI) u otros indicios de calidad. La calidad no sólo interesa a los editores de las revistas sino también a los investigadores por su repercusión en la evaluación de su actividad científica y de sus Instituciones. Se busca publicar en una revista de impacto para dar mayor visibilidad al artículo e institución o universidad del autor, porque son referencias básicas para la Agencia Nacional de Evaluación de la Calidad y Acreditación (ANECA) y para atraer recursos económicos al centro / universidad.

El $\mathrm{Fl}$ es un indicador bibliométrico que refleja el número de citas de artículos publicados en los últimos dos años por una revista (WoS). Divide las revistas en grupos que corresponden a áreas de investigación diferentes.

Para seleccionar la revista adecuada, se revisa el ámbito de las revistas de la misma cobertura temática y se elabora un listado de

\footnotetext{
${ }^{1}$ Puede verse como referencia el artículo de Torres Salinas (2013), Cómo publicar en revistas científicas de impacto: consejos y reglas sobre publicación científica https://ec3metrics.com/wp-content/uploads/2013/07/2-
}

aquellas en las que se podría publicar. A continuación se clasifican por orden de preferencia. Se tiene en cuenta el $\mathrm{Fl}$ pero también la evolución que presenta este índice, tratando de evitar revistas que presenten tendencias negativas.

En primer lugar se busca en la Web of Science (WoS) revistas del tema elegido, en la colección principal de WoS (Colección principal de Web of Science: índices de citas). Los resultados se ordenan por "veces citado" y se pueden limitar en el menú de la izquierda por "categorías del WoS» por tipo de documentos (por ejemplo artículos).

En Scopus realizaremos el mismo proceso, buscaremos por el tema de la investigación y se ordena por «citado por». Las posibilidades de limitación se encuentran en el menú izquierdo, por ejemplo especificando más el área temática o tipo de documento. También se puede realizar esta misma búsqueda en las bases de datos específicas del área temática a la que pertenezca la investigación.

El Fl de una revista concreta que se haya localizado se encuentra en varias fuentes. El Journal Citation Reports (JCR) es el índice bibliométrico más importante en la actualidad que recoge las mejores revistas científicas del mundo de la WoS. Para buscar el FI de una revista en JCR se selecciona la edición, ciencias o ciencias sociales, y el año. No existe $J C R$ para Arte y Humanidades a pesar de existir en WoS la base de datos Arts and $\mathrm{Hu}$ manities Citations Index. Se busca por el título de una revista específica y de esta forma se ve el $F I$, la tendencia del $F I$ y otras revistas relacionadas. En $J C R$ veremos también otros indicadores bibliométricos como el «índice de inmediatez», etc.

Basado en la base de datos Scopus (Elsevier), podemos ver SCImago Journal \& Country Rank $(S J R)^{2}$. Es el segundo índice bibliométrico internacional de importancia, competencia del JCR. En este caso se elige "Journal Search» y se busca una revista en concreto. Presenta tres indicadores, SJR, citas por documento y total de citas. La tendencia del indicador SJR debe ser positiva, evitando revistas que presenten una tendencia negativa.

ART\%C3\%8DCULO-C\%C3\%B3mo-Publicar-en-

Revistas-Cient\%C3\%ADficas-de-Impacto_Consejos-y-

Reglas-sobre-Publicaci\%C3\%B3n-Cient\%C3\%ADfica.pdf

${ }^{2}$ http://www.scimagojr.com/journalsearch.php 
Ulrich es otra fuente de información para buscar indicios de calidad. Recoge más de 300.000 publicaciones periódicas de 900 áreas temáticas: publicaciones académicas y de investigación, revistas electrónicas, títulos revisados por pares, revistas de gran popularidad. En la información sobre cada revista aparece si está evaluada por pares.

Latindex por su parte recoge revistas de investigación científica, que se editan en los países de América Latina, el Caribe, España y Portugal. Si la revista en cuestión aparece en "Catálogo» es que cumple los criterios de calidad y en "Características cumplidas» se indica el número de criterios cumplidos y cuáles no.

Las bibliotecas universitarias ofrecen guías con tutoriales sobre estos temas. Este es el caso de la Biblioteca de la Universidad de Sevilla que ofrece listados de las principales revistas por áreas, en el primer y segundo cuartil, las revistas españolas con $F /$ e índice $h$ y el índice $h 5$ y mediana $h 5$ del Google Scholar Metrics ${ }^{3}$.

Para seleccionar las revistas «TOP» del área podemos comenzar con JCR. Se selecciona el área específica y año y la opción de ver un grupo de revistas por categoría temática. Se selecciona el área de conocimiento (se pueden seleccionar varias) y se ven los datos de las revistas por factor impacto. Para seleccionar las revistas "TOP» en SCImago Journal \& Country Rank, se selecciona "Journal Rankings» y se elige el área y categoría temática y se ordenan por SJR.

Scopus ofrece la herramienta "Compare Journals» para evaluar el rendimiento de una revista científica. Para cada una proporciona gráficos que informan sobre la evolución del número de citas recibidas cada año, el número de artículos publicados en un período de tiempo o el número total de citas dividido por el número total de artículos publicados. Otros indicadores de calidad que ofrece esta herramienta se explicarán más adelante. Se pueden comparar hasta diez revistas de forma simultánea y pueden buscarse por título, ISSN o editor y limitar a un área concreta. Los resultados de las diferentes revistas aparecen en el mismo gráfico en distintos colores para facilitar su comparación.
Otro aspecto de interés son las fuentes en las que publican los investigadores reconocidos del área de interés. Para conocerlas una opción es buscar por autores en las distintas bases de datos que hemos mencionado, observando los títulos de las revistas en las que publican y para cada artículo, las revistas en las que publican los autores que los citan.

\section{FACTOR DE IMPACTO E INDICADORES BIBLIOMÉTRICOS} Con las métricas e indicadores actuales no es posible conocer de forma completa la calidad de las publicaciones científicas. No obstante existen indicadores cuantitativos que tradicionalmente han servido para valorar de forma relativa el impacto en la comunidad científica y han sido y siguen siendo de utilidad para el personal docente e investigador en los procesos de acreditación y sexenios principalmente.

Hasta ahora, el impacto de una revista se ha medido por las citas que recibe y la difusión en bases de datos de relevancia en su área con representación internacional ${ }^{4}$. Además se crearon una serie de índices bibliométricos que evalúan la trascendencia de las revistas, incluyendo las nacionales, que concentran las publicaciones de gran parte de nuestros investigadores.

Para evaluar las publicaciones científicas se utilizan una serie de criterios o indicadores que se aplican a distintos niveles. Tenemos por un lado las métricas aplicadas a los artículos en sí como contribución individual, por otro las aplicadas a revistas científicas o publicaciones, las aplicadas a los autores y por último a las instituciones, países o áreas temáticas. No todos valen para todo.

\section{NIVEL DE MÉTRICAS DE CONTRIBUCIÓN CIENTÍFICA} INDIVIDUAL

La contribución individual se identifica de forma generalizada con el artículo científico y es a este grupo de contribuciones al que se aplica la métrica más tradicional, el número de veces que un artículo se cita en determinado conjunto de revistas.

Técnicamente esta sección tiene sólo una métrica, las veces que se ha citado un artícu-

\footnotetext{
${ }^{3}$ http://bib.us.es/estudia_e_investiga/investigacion/estrateg ias/dondepublicar

Universidad de Oviedo dedicado a investigación. http://www.infobiblio.es/metrica-y-evaluaciones-

${ }^{4}$ Blog de Luisa Álvarez de Toledo de la Biblioteca de la cientificas-indicadores-y-sitios/
} 
lo, aunque veremos otros indicadores que han aparecido más recientemente. Actualmente son tres las mejores fuentes para contabilizar el número de citas y descubrir las conexiones de las citas entre artículos: WoS, Scopus y Google Académico. Los estudios han demostrado que el solapamiento entre estas tres fuentes depende de la disciplina [2].

Junto a estas métricas tradicionales, hay nuevas formas de medir el impacto de la producción científica como vimos en el primer artículo de esta serie. Estas otras métricas pretenden mostrar la presencia e impacto de la producción en los medios sociales y otras formas de participación social online como vistas, descargas o favoritos. Son las Ilamadas "Altmetrics», métricas complementarias que no alternativas de las tradicionales que ofrecen WoS o Scopus, entre otros. Las métricas altmétricas para este nivel podrían ser por un lado el número de menciones, citas, «Me Gusta», veces visto y descargas y por otro el tamaño de la audiencia o las evaluaciones formales o informales [2]. Para cada métrica veremos en la siguiente tabla su cobertura y características más significativas (Tabla 1).

Tabla 1. Métricas a nivel de artículo.

\begin{tabular}{|c|c|c|}
\hline Métrica & Cobertura & Características \\
\hline \multirow[t]{3}{*}{ Veces citado } & $\begin{array}{l}\text { Artículos de revistas en } \\
\text { WoS }\end{array}$ & Citas en y por artículos de revistas de WoS \\
\hline & $\begin{array}{l}\text { Artículos de revistas en } \\
\text { Scopus }\end{array}$ & Citas en y por artículos de revistas de Scopus \\
\hline & $\begin{array}{l}\text { Todo tipo de producción en } \\
\text { Google Académico }\end{array}$ & Citas en y por variedad de trabajos incluso no publicados \\
\hline $\begin{array}{l}\text { Article me- } \\
\text { trics }\end{array}$ & Artículos en Scopus & $\begin{array}{l}\text { Variedad de fuentes: lectores en Mendeley, impacto medio de la cita } \\
\text { por disciplina, apariciones en Wikipedia, tuits o post en blogs, etc. }\end{array}$ \\
\hline $\begin{array}{l}\text { Usage } \\
\text { counts }\end{array}$ & $\begin{array}{l}\text { Artículos en Web of Scien- } \\
\text { ce }\end{array}$ & $\begin{array}{l}\text { Uso de los documentos en WoS en los últimos } 180 \text { días o desde } \\
2013\end{array}$ \\
\hline ALM-PLoS & Artículos en PLOS & $\begin{array}{l}\text { Uso en PLOS y PubMed Central } \\
\text { - Citas en PubMed Central, Scopus, ISI Web de Ciencias y } \\
\text { CrossRef. } \\
\text { - Capturas en CiteUlike, Mendeley, Google+, etc. } \\
\text { - Medios sociales: Twitter, Facebook, Blogs, etc. }\end{array}$ \\
\hline $\begin{array}{l}\text { Altmetric } \\
\text { Altme- } \\
\text { tric.com }\end{array}$ & $\begin{array}{l}\text { Artículos científicos } \\
\text { Cálculo del donuts de } \\
\text { Altmetric.com }{ }^{5}\end{array}$ & $\begin{array}{l}\text { - Uso en PubMed, Arxiv, Wiley, Taylor \& Francis, Springer, o DOI } \\
\text { - Capturas en Mendeley, etc. } \\
\text { - Medios sociales: Twitter, Facebook, Blogs, Wikipedia, etc. }\end{array}$ \\
\hline ImpactStory & $\begin{array}{l}\text { Todo tipo de productos de } \\
\text { investigación }\end{array}$ & $\begin{array}{l}\text { - Uso en PLOS, PubMed, Slideshare, Vimeo, Figshare, ORCID, etc. } \\
\text { - Citas en Scopus, PubMed, Google Académico, etc. } \\
\text { - Capturas en Mendeley, SlideShare, Delicious, etc. } \\
\text { - Medios sociales: Twitter, Facebook, Blogs, Wikipedia, SlideShare, } \\
\text { Delicious, etc. }\end{array}$ \\
\hline $\begin{array}{l}\text { Plum } X \text { de } \\
\text { Plum Analy- } \\
\text { tics (EBSCO) }\end{array}$ & $\begin{array}{l}\text { Todo tipo de productos } \\
\text { científicos } \\
\text { Cálculo de Plum } \mathrm{X}^{6}\end{array}$ & $\begin{array}{l}\text { - Uso en EBSCO, PLOS, Facebook, WorldCat, etc, } \\
\text { - Citas en Scopus, PubMed, etc. } \\
\text { - Capturas y Menciones en CiteUlike, Delicious, YouTube, Mendeley, } \\
\text { Wikipedia, etc. } \\
\text { - Medios sociales: Facebook, SlideShare, Google+, etc. }\end{array}$ \\
\hline
\end{tabular}

5 https://help.altmetric.com/support/solutions/articles/6000060969-how-is-the-altmetric-score-calculated6 http://plumanalytics.com/learn/about-metrics/ 
Tradicionalmente los dos grandes recursos WoS y Scopus reducen las citas a artículos o realizadas por artículos de revistas a pesar de la existencia de otros materiales citables en la WoS. Este nivel de métricas podría incluir también las contribuciones individuales como posts en blogs o capítulos de libros, blogs, actas de congresos, posters, infografías, patentes o cualquier otro tipo de contribución individual. Google Académico (GA) sin embargo contempla una gran variedad de productos como trabajos no publicados, tesis, presentaciones, conferencias o patentes.

Scopus indicaba en su blog $^{7}$ que una sola métrica no puede dar la visión completa del impacto y repercusión de la producción científica. Por esta razón, lanzó en julio de 2015 un nuevo producto de métricas a nivel de artículo que aunaba las aportaciones de las métricas tradicionales con las altmétricas para dar una visión del impacto y engagement desde distintas fuentes. De esta forma, en la información detallada del artículo, en la barra lateral se ofrece un número mínimo de métricas significativas para el investigador que evalúan tanto el impacto de las citas como el nivel de engagement de la comunidad. Se ofrecen datos cuando los hay de lectores en Mendeley, impacto medio de la cita por disciplina, apariciones en Wikipedia, twits o post en blogs, entre otros. Con el enlace "View all metrics», se accede al módulo completo de "Article Metrics» donde se pueden encontrar todas las métricas y contenidos destacados. Como puede observarse, se están dando pasos acelerados para que las métricas procedentes de fuentes distintas de las tradicionales, sean tenidas en cuenta en la valoración de la producción científica y en su recomendación como recursos de referencia.

También la Web of Science se ha sumado a esta corriente de las altmétricas con un nuevo indicador "usage counts» que mide el uso de los documentos en los últimos 180 días o desde 2013. Es de interés para conocer la atención de los investigadores hacia aquellos artículos que no han sido citados.

Cada vez más editores ofrecen estas altmétricas en sus productos, BioMed Central, Nature Publishing Group, Springer, Wiley o Taylor \& Francis Online. Los proveedores más conocidos de métricas altmétricas son Altmetric.com, ImpactStory, Plum X y PLOS.

MÉTRICAS USADAS PARA EVALUAR EL IMPACTO DE LA PUBLICACIÓN

Las métricas científicas sirven para conocer qué publicaciones son las mejores en el ámbito científico de interés. Para un investigador la calidad de la revista es un factor muy importante para saber dónde es mejor publicar, qué valoración científica tiene un determinado investigador, una institución, un grupo científico y cuáles son las tendencias de investigación.

Las acreditaciones de la ANECA (Agencia Nacional de Evaluación de la Calidad y Acreditación y los criterios de calidad y acreditación que ahora incluye subcomisión académica -CNEAl-) obligan a los investigadores españoles a conocer el funcionamiento de estas valoraciones e indicadores.

Junto a las métricas tradicionales podemos observar también las altmétricas que van apareciendo para medir a nivel de publicación. En la tabla que sigue veremos una descripción de cada métrica con sus características y observaciones de su uso (Tabla 2).

El Fl del JCR es uno de los índices más conocidos, pero hay más formas de medir la calidad científica. En el caso de España los investigadores pueden utilizar otros repertorios y bases de datos además de los Journal Citation Reports para sus evaluaciones de calidad. La aparición de Scopus en 2004 obligó a WoS a competir y crear bases de datos para tipologías diferentes a las revistas: Book Citation Index y Data Citation Index, incluidos en su Core Collection.

También dentro del Core Collection, Emerging Source Citation Index (ESCI, 2015), incluye 56.000 revistas en proceso de evaluación en las que predominan los trabajos en inglés $(81 \%)$, en español sólo el $7,8 \%$.

Fuera de Core Collection incluye bases de datos como SciELO Citation Index para Latinoamérica pero ni SciELO ni ESCl se incluyen en la elaboración de indicadores de impacto o cobertura del JCR.

7 http://blog.scopus.com/posts/new-scopus-article-metricsa-better-way-to-benchmark-articles 
Tabla 2. Métricas para el impacto de las publicaciones.

\begin{tabular}{|c|c|c|}
\hline Métrica & Características & Observaciones \\
\hline $\begin{array}{l}\text { Factor de impacto } \\
\text { JCR de WoS } \\
\text { Thomson-Reuters }\end{array}$ & $\begin{array}{l}\text { Factor de impacto de la } \\
\text { publicación: "N de citas } \\
\text { recibidas en el año actual de } \\
\text { las publicaciones de la } \\
\text { revista en los dos años } \\
\text { anteriores y dividido por el } \\
\text { número de artículos publica- } \\
\text { dos por la revista en esos } \\
\text { dos años anteriores". } \\
\text { En Arte y Humanidades no } \\
\text { existen Factores de Impacto } \\
\text { (salvo excepciones: historia, } \\
\text { lingüística,...). }\end{array}$ & $\begin{array}{l}\text { Es un indicador que permite comparar revistas y evaluar } \\
\text { la importancia relativa de una revista dentro de un mismo } \\
\text { campo científico. } \\
\text { Para DORA (San Francisco Declaration on Research } \\
\text { Assessment): } \\
\text { - No es representativo del índice de citación de los artícu- } \\
\text { los. } \\
\text { - Es variable según la disciplina científica. } \\
\text { - Es sensible al número y el tipo de trabajos de las revis- } \\
\text { tas. } \\
\text { - Puede manipularse por parte de los editores } \\
\text { - Existe un problema con las autocitas. } \\
\text { - La ventana de citación de dos años a veces no es } \\
\text { suficiente. } \\
\text { - Imposibilidad de reflejar el impacto de un artículo en } \\
\text { particular. } \\
\text { - Disciplinas con distintos modelos de citas, dificulta la } \\
\text { comparación entre campos. No hay para Humanidades y } \\
\text { es escaso el número de revistas de lengua no inglesa. } \\
\text { Valdrían simplemente las revistas indexadas en el A\&HCl } \\
\text { de Thomson Reuters. }\end{array}$ \\
\hline $\begin{array}{l}\text { Eigenfactor score } \\
\text { WoS (Thomson Reu- } \\
\text { ters) } \\
\text { (Eigenfactor.org) }\end{array}$ & $\begin{array}{l}\text { Desarrollado por la Univer- } \\
\text { sidad de Washington, sobre } \\
\text { la importancia de revistas } \\
\text { científicas basados en la } \\
\text { información de citas del } \\
\text { Journal Citation Reports. } \\
\text { Ordena las revistas por citas } \\
\text { con un algoritmo parecido al } \\
\text { de las páginas webs de } \\
\text { Google. }\end{array}$ & $\begin{array}{l}\text { Índice basado en el número de veces que los artículos } \\
\text { publicados en los cinco años pasados han sido citados en } \\
\text { el presente año. Esta puntuación está influenciada por el } \\
\text { tamaño de una publicación medida por el número de } \\
\text { artículos anuales publicados (una revista que duplica el } \\
\text { número de artículos que publica, duplica igualmente su } \\
\text { puntuación Eigenfactor). } \\
\text { Diferencia esencial con JCR: } \\
\text { - Número de años. } \\
\text { - Se cuentan las citas tanto de ciencias como de ciencias } \\
\text { sociales. } \\
\text { - Elimina las autocitas (citas dentro de la misma revista). } \\
\text { - Da más valor a las citas aparecidas en las revistas que } \\
\text { tienen mayor cantidad de consultas y citas. }\end{array}$ \\
\hline Article Influence & $\begin{array}{l}\text { Desarrollado por la Univer- } \\
\text { sidad de Washington. }\end{array}$ & $\begin{array}{l}\text { Mide la media de la influencia de los artículos de una } \\
\text { publicación, por lo que es comparable al factor de impacto } \\
\text { del JCR. }\end{array}$ \\
\hline $\begin{array}{l}\text { SJR Scimago Journal } \\
\text { Rank } \\
\text { del grupo SCImago Lab } \\
\text { Datos de Scopus } \\
\text { (Elsevier) }\end{array}$ & $\begin{array}{l}\text { Refleja el prestigio de la } \\
\text { revista mediante el número } \\
\text { de citas ponderadas por } \\
\text { documento. } \\
\text { Índice de impacto semejante } \\
\text { al JCR pero elaborado a } \\
\text { partir de la base de datos de } \\
\text { SCOPUS. }\end{array}$ & $\begin{array}{l}\text { Analiza las citas durante un período de tres años de } \\
16.000 \text { revistas. Está construido como una variación del } \\
\text { Eigenfactor, y se inspira en el PageRank de Google para } \\
\text { evaluar el impacto de una publicación combinando el } \\
\text { número de citas recibidas con la influencia de las publica- } \\
\text { ciones que la citan. El área de investigación, calidad y y } \\
\text { reputación de la revista científica tienen un impacto direc- } \\
\text { to sobre el valor de la cita. Por ello, la cita de una revista } \\
\text { con un alto SJR vale más que la cita en una revista con } \\
\text { un SJR más bajo. } \\
\text { Se calcula en http://www.scimagojr.com/. }\end{array}$ \\
\hline $\begin{array}{l}\text { Impacto por publicación } \\
\text { al año (IPP) }\end{array}$ & $\begin{array}{l}\text { Creado por la Universidad } \\
\text { de Leiden. } \\
\text { Mide la ratio de citas por } \\
\text { artículo publicado en la } \\
\text { revista (media de citas de } \\
\text { los artículos). }\end{array}$ & $\begin{array}{l}\text { Indicador similar al FI de Thomson Reuters (sólo } 2 \text { años). } \\
\text { Se puede consultar en Scopus, Compare Journals y en } \\
\text { Journal Metrics (http://www.journalmetrics.com/). }\end{array}$ \\
\hline
\end{tabular}


Tabla 2 (continuación). Métricas para el impacto de las publicaciones.

\begin{tabular}{|c|c|c|}
\hline Métrica & Características & Observaciones \\
\hline $\begin{array}{l}\text { Impacto de la fuente } \\
\text { normalizada por artícu- } \\
\text { lo. Source Normalized } \\
\text { Impact per Paper } \\
\text { (SNIP) }\end{array}$ & $\begin{array}{l}\text { Creado por la Universidad } \\
\text { de Leiden. } \\
\text { Mide el impacto de una cita } \\
\text { según las características de } \\
\text { la materia sobre la que se } \\
\text { investiga. } \\
\text { Se basa en la comparación } \\
\text { de publicaciones dentro de } \\
\text { sus campos temáticos, } \\
\text { contabilizando la frecuencia } \\
\text { con la que los autores citan } \\
\text { otros documentos, y la } \\
\text { inmediatez del impacto de la } \\
\text { cita. }\end{array}$ & $\begin{array}{l}\text { Es más objetivo que JCR. Se basa en Scopus y hace } \\
\text { referencia a tres años y no a dos como el JCR. } \\
\text { Tiene en cuenta el área de investigación corrigiendo las } \\
\text { diferencias en la probabilidad de ser citado que existe } \\
\text { entre revistas de distintas materias e incluso entre revis- } \\
\text { tas del mismo área de conocimiento. } \\
\text { En un campo de investigación con una menor frecuencia } \\
\text { de citas, cada cita tiene un valor superior al de las citas } \\
\text { en campos con mayor frecuencia de citas. } \\
\text { Si la revista en cuestión tiene un SNIP que cambia fre- } \\
\text { cuentemente, indica que puede variar en calidad. } \\
\text { (http://www.journalindicators.com/indicators) }\end{array}$ \\
\hline $\begin{array}{l}\text { Índice de inmediatez } \\
\text { para revistas científicas }\end{array}$ & $\begin{array}{l}\text { Mide el número de artículos } \\
\text { publicados en un año y } \\
\text { citados ese mismo año. Se } \\
\text { pueden analizar en WoS, } \\
\text { Scopus, Google Académico }\end{array}$ & $\begin{array}{l}\text { Mide la rapidez con la que se citan los artículos de una } \\
\text { revista científica y permite identificar revistas líderes en } \\
\text { investigaciones de amplia repercusión. } \\
\text { Factor de Impacto Potencial de las Revistas Médicas } \\
\text { Españolas (2001-2005) } \\
\text { (http://ime.uv.es/imecitas/impacto_ime.asp?anyo=4) }\end{array}$ \\
\hline $\begin{array}{l}\text { Cuartil. Se puede ver } \\
\text { en } \\
\text { JCR, SJR y WoS }\end{array}$ & $\begin{array}{l}\text { Indicador o medida de posi- } \\
\text { ción de una revista en rela- } \\
\text { ción con todas las de su } \\
\text { área. }\end{array}$ & $\begin{array}{l}\text { Si dividimos en } 4 \text { partes iguales un listado de revistas } \\
\text { ordenadas de mayor a menor factor de impacto, cada una } \\
\text { de estas partes será un cuartil. Las revistas con el factor } \\
\text { de impacto más alto estarán en el primer cuartil. } \\
\text { Puede consultarse en Journal Citation Reports (JCR), } \\
\text { SClmago Journal \& Country Rank } \\
\text { (http://www.scimagojr.com/) y Web of Science. }\end{array}$ \\
\hline $\begin{array}{l}\text { Presencia en Master } \\
\text { List de la Web of } \\
\text { Science }\end{array}$ & $\begin{array}{l}\text { Si la revista no tiene FI hay } \\
\text { que consultar el master list } \\
\text { para saber si está en la base } \\
\text { de datos de WoS. }\end{array}$ & $\begin{array}{l}\text { Dirección URL (http://ip-science.thomsonreuters.com/cgi- } \\
\text { bin/jrnlst/jloptions.cgi?PC=master) }\end{array}$ \\
\hline $\begin{array}{l}\text { ESCI } \\
\text { Emerging Source Cita- } \\
\text { tion Index }\end{array}$ & $\begin{array}{l}\text { Si no aparece en WoS hay } \\
\text { que consultar ESCI que } \\
\text { ofrece la posibilidad de } \\
\text { entrar en WoS. }\end{array}$ & $\begin{array}{l}\text { Listado de revistas emergentes de la Biblioteca de la } \\
\text { Universidad } \\
\text { (http://bib.us.es/sites/bib3.us.es/files/emerging-source- } \\
\text { citation-index.xlsx) }\end{array}$ \\
\hline $\begin{array}{l}\text { Publishers Scholar } \\
\text { Metrics } \\
\text { EC3 } \\
\text { (http://www.publishers- } \\
\text { scholarmetrics.info/) }\end{array}$ & $\begin{array}{l}\text { Mide el impacto de las } \\
\text { editoriales de monografías } \\
\text { científicas. }\end{array}$ & $\begin{array}{l}\text { Ámbito de las Humanidades y Ciencias Sociales. } \\
\text { Mide el impacto a partir del total de citas de libros publi- } \\
\text { cados por docentes e investigadores de las Universidades } \\
\text { públicas españolas, indizados en Google Académico } \\
\text { hasta } 2012 \text {. }\end{array}$ \\
\hline $\begin{array}{l}\text { SPI (Scholarly Publish- } \\
\text { ers Indicators) } \\
\text { CSIC } \\
\text { (http://ilia.cchs.csic.es/ } \\
\text { SPI/) }\end{array}$ & $\begin{array}{l}\text { Indicadores de calidad para } \\
\text { libros y editoriales de carác- } \\
\text { ter científico. }\end{array}$ & $\begin{array}{l}\text { Ámbito de las Humanidades y Ciencias Sociales. Muestra } \\
\text { un ranking de editoriales basado en la opinión de exper- } \\
\text { tos españoles en dichas áreas, de forma general para } \\
\text { todas las áreas y especializado por disciplinas. }\end{array}$ \\
\hline $\begin{array}{l}\text { Book Citation Index } \\
\text { de Thomson }\end{array}$ & $\begin{array}{l}\text { Es un indicador de calidad } \\
\text { para la evaluación de libros } \\
\text { y editores académicos. }\end{array}$ & $\begin{array}{l}\text { Está enfocado al mundo anglosajón, con gran concentra- } \\
\text { ción editorial (solo } 18 \text { constituyen la base de datos). No } \\
\text { hay editoriales universitarias y poca presencia de las } \\
\text { Ciencias Sociales y Humanidades. }\end{array}$ \\
\hline
\end{tabular}


Tabla 2 (continuación). Métricas para el impacto de las publicaciones.

\begin{tabular}{|c|c|c|}
\hline Métrica & Características & Observaciones \\
\hline $\begin{array}{l}\text { Índice h5 de las revis- } \\
\text { tas y otras fuentes } \\
\text { documentales de Goo- } \\
\text { gle Scholar Metrics }\end{array}$ & $\begin{array}{l}\text { Aparecen las revistas inclui- } \\
\text { das en Google Scholar que } \\
\text { han publicado al menos } 100 \\
\text { artículos y cuentan con } \\
\text { alguna cita. }\end{array}$ & $\begin{array}{l}\text { Pulsando en el índice h5 (de los últimos cinco años com- } \\
\text { pletos) de cada revista, se pueden ver los artículos más } \\
\text { citados y quién los ha citado. } \\
\text { La mediana h5 de una publicación es el número mediano } \\
\text { de citas de los artículos que componen el índice h5. } \\
\text { Relación de publicaciones en Google Scholar Metrics } \\
\text { (https://scholar.google.com/citations) }\end{array}$ \\
\hline $\begin{array}{l}\text { ERIH PLUS } \\
\text { (https://dbh.nsd.uib.no/ } \\
\text { publisering- } \\
\text { skanaler/erihplus/) }\end{array}$ & $\begin{array}{l}\text { European Reference Index } \\
\text { for the Humanities }\end{array}$ & $\begin{array}{l}\text { Su objetivo es mejorar la visibilidad de investigación de } \\
\text { calidad en humanidades y ciencias sociales en Europa, y } \\
\text { facilitar acceso a revistas de investigación publicadas en } \\
\text { todas las lenguas europeas. }\end{array}$ \\
\hline $\begin{array}{l}\text { Clasificación Integrada } \\
\text { de Revistas Científicas } \\
\text { de sociales y humanas } \\
\text { - CIRC }\end{array}$ & & $\begin{array}{l}\text { Clasificación en función de su calidad integrando los } \\
\text { productos de evaluación existentes considerados positi- } \\
\text { vamente por las diferentes agencias de evaluación nacio- } \\
\text { nales como ANECA. }\end{array}$ \\
\hline $\begin{array}{l}\text { Co-author Index } \\
\text { (http://www.coauthorind } \\
\text { ex.info/) }\end{array}$ & $\begin{array}{l}\text { Mide el índice de coautoría } \\
\text { de artículos publicados por } \\
\text { autores españoles en revis- } \\
\text { tas nacionales e internacio- } \\
\text { nales. } \\
\text { Elaborado por el grupo de } \\
\text { investigación EC3. }\end{array}$ & $\begin{array}{l}\text { Hábitos y prácticas de coautoría de los investigadores } \\
\text { españoles en los distintos campos y disciplinas en los } \\
\text { artículos publicados en } 20.000 \text { revistas. } \\
\text { Se pueden consultar por revista o por disciplina para un } \\
\text { año concreto. } \\
\text { Datos a nivel global para todo el período analizado y su } \\
\text { evolución a lo largo del tiempo. } \\
\text { En el caso de las disciplinas se muestran los valores de } \\
\text { las revistas nacionales y las internacionales (las revistas } \\
\text { extranjeras procesadas en la Web of Science), para poder } \\
\text { comparar en cada año las diferencias en los índices de } \\
\text { coautoría en uno y otro ámbito. }\end{array}$ \\
\hline $\begin{array}{l}\text { TIF Factor de impacto } \\
\text { de Twitter de la publi- } \\
\text { cación [1]. } \\
\text { Métrica alternativa }\end{array}$ & $\begin{array}{l}\text { Mide la influencia de una } \\
\text { revista en los medios socia- } \\
\text { les. Elaborado a partir de } \\
\text { seguidores, twits y RT du- } \\
\text { rante dos años. }\end{array}$ & $\begin{array}{l}\text { EI TIF se calcula por el número de RT que tienen cada } \\
\text { twit original y relevante. } \\
\text { Estudio elaborado en el área de Urología. }\end{array}$ \\
\hline $\begin{array}{l}\text { Altmetric } \\
\text { Impacto de la publica- } \\
\text { ción } \\
\text { Métrica alternativa }\end{array}$ & $\begin{array}{l}\text { Elaborado por Altmetric.com } \\
\text { para artículos }\end{array}$ & $\begin{array}{l}\text { Con restricciones. Las métricas para revistas se basan en } \\
\text { los datos altmétricos de los artículos que incluyen. }\end{array}$ \\
\hline $\begin{array}{l}\text { Altmetrics impacto de } \\
\text { libros y capítulos de } \\
\text { libros } \\
\text { Métrica alternativa }\end{array}$ & $\begin{array}{l}\text { Elaborado por Altmetric.com } \\
\text { para libros y capítulos de } \\
\text { libros. }\end{array}$ & \\
\hline
\end{tabular}
MÉTRICAS USADAS PARA EVALUAR EL IMPACTO
DE LOS AUTORES

Estas métricas consideran el impacto de un autor individual a lo largo de su carrera. En el siguiente cuadro veremos cada métrica, sus características y observaciones más relevantes (Tabla 3).
MÉTRICAS USADAS PARA EVALUAR EL IMPACTO DE LAS INSTITUCIONES

Estas métricas representan una tendencia importante en la bibliometría y las altmétricas por la creciente necesidad de justificar y cuantificar el impacto de la actividad científica de las instituciones, por ejemplo, para temas 
de financiación. Aparecen así herramientas para medir el impacto de las publicaciones de los miembros de la organización. En la tabla que sigue se presentan las métricas, características y observaciones que consideramos necesarias (Tabla 4).

Tabla 3. Métricas a nivel de autor.

\begin{tabular}{|c|c|c|}
\hline Métrica & Características & Observaciones \\
\hline $\begin{array}{l}\text { Índice h de } \\
\text { Hirsch } \\
\text { En WoS y Sco- } \\
\text { pus } \\
\text { (https://www.sco } \\
\text { pus.com/search/ } \\
\text { form/authorFree } \\
\text { Lookup.uri ) }\end{array}$ & $\begin{array}{l}\text { El índice varía según la fuente. Se } \\
\text { utiliza para valorar a los investiga- } \\
\text { dores, áreas temáticas, institucio- } \\
\text { nes, etc. Analiza el número de } \\
\text { publicaciones citadas y el número } \\
\text { de citas que han tenido. Un cientí- } \\
\text { fico tiene índice h si ha publicado } \\
\text { h trabajos con al menos h citas } \\
\text { cada uno. }\end{array}$ & $\begin{array}{l}\text { Es útil para detectar los investigadores más destacados de } \\
\text { un área de conocimiento. } \\
\text { Se puede calcular para un autor, varios autores y varias } \\
\text { publicaciones. } \\
\text { Las autocitas se excluyen. } \\
\text { Depende demasiado de los años de investigación. } \\
\text { Se centra en los artículos pero no en otro tipo de materia- } \\
\text { les. Sólo tiene en cuenta las citas y artículos en las revistas } \\
\text { de WoS. }\end{array}$ \\
\hline $\begin{array}{l}\text { Índice } \mathrm{h} \text { de } \\
\text { Google Acadé- } \\
\text { mico }\end{array}$ & $\begin{array}{l}\text { Índice bibliométrico que indica que } \\
\mathrm{h} \text { publicaciones se han citado al } \\
\text { menos } \mathrm{h} \text { veces en Google aca- } \\
\text { démico. }\end{array}$ & $\begin{array}{l}\text { Con la aplicación Mis Citas un autor puede crear su perfil } \\
\text { en Google Académico y acceder a estos índices. } \\
\text { Se consulta también en H-Index Scholar }{ }^{8} \text {, producto que } \\
\text { dejó de actualizarse en } 2013 \text {, y está elaborado por el Grupo } \\
\text { EC3, para medir el rendimiento de la producción académica } \\
\text { de profesores e investigadores de universidades públicas } \\
\text { españolas de Humanidades y Ciencias Sociales, a partir } \\
\text { del recuento de sus publicaciones y de las citas bibliográfi- } \\
\text { cas que han recibido a través de Google Académico. }\end{array}$ \\
\hline $\begin{array}{l}\text { Índice } \mathrm{g} \text { de } \\
\text { Google Acadé- } \\
\text { mico }\end{array}$ & $\begin{array}{l}\text { Cuantifica la productividad biblio- } \\
\text { métrica basada en el historial de } \\
\text { publicaciones de los autores. }\end{array}$ & $\begin{array}{l}\text { Es semejante al índice } \mathrm{h} \text {, pero da más relevancia a los } \\
\text { artículos que tienen un alto número de citas. Centra el peso } \\
\text { en los trabajos académicos más citados. } \\
\text { Índice } \mathrm{G}=15 \text { (la cantidad de citas acumuladas por estos } 15 \\
\text { artículos más citados es superior a } 15 \text { al cuadrado). } \\
\text { Se consulta tambien en H-Index Scholar de EC3. }\end{array}$ \\
\hline $\begin{array}{l}\text { Índice i10 } \\
\text { Google acadé- } \\
\text { mico }\end{array}$ & $\begin{array}{l}\text { Toma el número de artículos que } \\
\text { un autor ha publicado hasta la } \\
\text { fecha y calcula cuántos de ellos } \\
\text { han generado al menos } 10 \text { citas. }\end{array}$ & $\begin{array}{l}\text { Es de gran simplicidad. Pero no cuenta toda la historia del } \\
\text { impacto del autor, tiene que verse en relación con el índice } \\
\text { h. }\end{array}$ \\
\hline $\begin{array}{l}\text { Publish or } \mathrm{Pe}- \\
\text { rish }\end{array}$ & $\begin{array}{l}\text { Aplicación para evidenciar el } \\
\text { impacto de las publicaciones de } \\
\text { un autor que no están en JCR. }\end{array}$ & $\begin{array}{l}\text { Analiza una gran cantidad de factores de calidad a partir de } \\
\text { los datos obtenidos en Google Scholar y Microsoft Acade- } \\
\text { mic Search. Las métricas que ofrece son: índice h, índice g, } \\
\text { índice h individual y por grupos, media de crecimiento de } \\
\text { los índices, etc. } \\
\text { Programa gratuito que hay que descargar } \\
\text { (http://www.harzing.com/pop.htm). }\end{array}$ \\
\hline $\begin{array}{l}\text { Scholarometer } \\
\text { (http://scholaro } \\
\text { me- } \\
\text { ter.indiana.edu/) }\end{array}$ & $\begin{array}{l}\text { Extensión del navegador que } \\
\text { ofrece una interfaz interactiva de } \\
\text { Google Académico. }\end{array}$ & $\begin{array}{l}\text { Ofrece el índice } \mathrm{h} \text { y porcentajes propios de la posición del } \\
\text { autor en su área de trabajo. }\end{array}$ \\
\hline
\end{tabular}

8 http://hindexscholar.com/ 
Tabla 4. Métricas a nivel de instituciones

\begin{tabular}{|c|c|c|}
\hline Métricas & Características & Observaciones \\
\hline $\begin{array}{l}\text { Essential } \\
\text { Indicators } \text { Rance } \\
\text { ings (ESI Thomson } \\
\text { and Reuters) }\end{array}$ & $\begin{array}{l}\text { Proporciona los principales } \\
\text { indicadores científicos por } \\
\text { países, instituciones, investi- } \\
\text { gadores más importantes y } \\
\text { sus publicaciones, principales } \\
\text { áreas de investigación. Se } \\
\text { puede consultar WoS } \\
\text { Cubre } 8.500 \text { publicaciones de } \\
\text { todo el mundo. }\end{array}$ & $\begin{array}{l}\text { El ranking institucional se basa en las publicaciones de los } \\
\text { afiliados a una institución y las citas que han recibido esas } \\
\text { publicaciones. } \\
\text { Este indicador se encuentra actualmente integrado en la } \\
\text { plataforma InCites. } \\
\text { Analiza el rendimiento de la investigación de empresas, } \\
\text { instituciones, naciones y publicaciones. } \\
\text { Identifica tendencias significativas en las ciencias y ciencias } \\
\text { sociales. Evalúa empleados, colaboradores, revisores y } \\
\text { coetáneos potenciales. Determina el resultado de una inves- } \\
\text { tigación y el impacto en campos de investigación específicos. }\end{array}$ \\
\hline $\begin{array}{l}\text { Snowball metrics } \\
\text { (http://www.snowb } \\
\text { allmetrics.com/) } \\
\text { Métricas Altmetrics }\end{array}$ & $\begin{array}{l}\text { Iniciativa nacida de la colabo- } \\
\text { ración entre } 8 \text { universidades } \\
\text { de UK y Elsevier para crear } \\
\text { un conjunto de metodologías } \\
\text { que sirvieran para comparar- } \\
\text { se las instituciones entre si } \\
\text { mapeando varias métricas } \\
\text { como recuento de citas, citas } \\
\text { por resultados, índice h, y } \\
\text { colaboración. Las altmetrics } \\
\text { también aparecen en este } \\
\text { informe. }\end{array}$ & $\begin{array}{l}\text { Contemplan } 12 \text { principios para uso responsable de las métri- } \\
\text { cas }^{9} \text {. } \\
\text { Cuatro categorías de métricas alternativas: } \\
\text { - Actividad científica: descargas y entradas en gestores } \\
\text { como Mendeley o CiteULike. } \\
\text { - Actividad social: menciones en medios sociales como } \\
\text { Twitter, Facebook and Google+. } \\
\text { - Comentarios científicos: revisiones, artículos de expertos y } \\
\text { científicos, en sitios como F1000 Prime, blogs de investiga- } \\
\text { ción y Wikipedia. } \\
\text { - Medios: cobertura de los resultados de la investigación en } \\
\text { los distintos medios (Altmetric.com). }\end{array}$ \\
\hline $\begin{array}{l}\text { SCImago Institu- } \\
\text { tions Rankings. } \\
\text { Datos de SCOPUS } \\
\text { (http://www.scimag } \\
\text { oir.com/) }\end{array}$ & $\begin{array}{l}\text { Herramienta gratuita online } \\
\text { que clasifica las instituciones } \\
\text { de investigación en todo el } \\
\text { mundo en base a tres carac- } \\
\text { terísticas: evaluación, innova- } \\
\text { ción y visibilidad web. }\end{array}$ & $\begin{array}{l}\text { Usa datos de Scopus para crear métricas para siete indica- } \\
\text { dores: resultados, talento científico, excelencia, liderazgo, } \\
\text { colaboración internacional, impacto normalizado, especiali- } \\
\text { zación. } \\
\text { Los usuarios pueden obtener los resultados según indicado- } \\
\text { res específicos. }\end{array}$ \\
\hline SciVal & Datos proceden de Scopus. & $\begin{array}{l}\text { Información de las citas de los documentos de los miembros } \\
\text { de una organización, estudios de datos cruzados por grupos } \\
\text { de investigación y personalizados. }\end{array}$ \\
\hline InCites & $\begin{array}{l}\text { Datos proceden de Web of } \\
\text { Science. Incluye ESI Ranking. }\end{array}$ & $\begin{array}{l}\text { Métricas a nivel de artículo para una institución. Los usuarios } \\
\text { pueden crear sus listas de autores o instituciones ordenadas } \\
\text { por distintos criterios como veces citados o media de citas } \\
\text { por documento. }\end{array}$ \\
\hline $\begin{array}{l}\text { Altmetric } \\
\text { Altmetric.com } \\
\text { Métricas Altmetrics }\end{array}$ & $\begin{array}{l}\text { Ofrece dos herramientas } \\
\text { analíticas para instituciones. }\end{array}$ & $\begin{array}{l}\text { Altmetrics for Institutions ofrece métricas detalladas por } \\
\text { artículo e institución, departamento o autor. } \\
\text { Altmetric Explorer para buscar una selección de revistas, } \\
\text { temas o búsquedas en PubMed y sus datos altmétricos. }\end{array}$ \\
\hline $\begin{array}{l}\text { PlumX de EBSCO } \\
\text { Métricas Altmetrics }\end{array}$ & $\begin{array}{l}\text { Ofrece cuatro métricas: uso, } \\
\text { capturas, citas y medios } \\
\text { sociales. }\end{array}$ & $\begin{array}{l}\text { Dentro de una institución, ofrece estas métricas a nivel de } \\
\text { facultad, departamento, grupo de investigación, revista insti- } \\
\text { tucional y personalizado. }\end{array}$ \\
\hline
\end{tabular}

9 Documento de Elsevier "Response to HEFCE's call for evidence: independent review of the role of metrics in research assessment" http://www.elsevier.com/research-intelligence/resource-library/response-to-hefces

(c) Ediciones Universidad de Salamanca / CC BY-NC-ND [160] Rev. ORL, 2016, 7, 3, pp. 151-162 
OTROS INDICIOS DE CALIDAD DE LAS PUBLICACIONES

Además de las fuentes y métricas que hemos visto, existen otros recursos en los que los investigadores pueden ver evidencias de calidad de sus publicaciones. Entre ellos encontramos $I n-R E C J^{10}$ e In-RECS ${ }^{11}$ para ciencias jurídicas y sociales respectivamente. Estos índices se dejaron de actualizar en 2014 pero puede consultarse la versión archivada en Internet Archive. Son índices bibliométricos elaborados por EC3 que analizan citas de revistas de estas áreas y determinan la relevancia, influencia e impacto científico de las revistas y autores que publican en las mismas y de las instituciones a las que pertenecen. Aportan el factor de impacto o cuartil.

Latindex ${ }^{12}$ es el "Sistema Regional de Información en Línea para Revistas Científicas de América Latina, el Caribe, España y Portugal». Reúne información bibliográfica sobre revistas y ofrece criterios de evaluación para revistas impresas y electrónicas. Para que una revista sea catalogada, debe pasar obligatoriamente los 8 criterios de «características básicas» y debe obtener al menos el $75 \%$ de todos los criterios juntos.

MIAR ${ }^{13}$ (Matriu d'Informació per l'Avaluació de Revistes) es una base de datos que establece anualmente la identificación y evaluación de revistas en las Ciencias Sociales y Humanidades asignándoles un ECDS. En la actualidad contiene más de 28.911 títulos.

$R E S H^{14}$ es un sistema de información que ofrece indicadores cualitativos y cuantitativos desarrollados por el Grupo de Investigación EPUC del CSIC y EC3 de la Universidad de Granada. Para cada revista indica qué criterios cumple o no según la ANECA y Latindex. Se trata de indicadores de calidad editorial y proporciona también el factor de impacto.

CIRC $2.0^{15}$ de EC3Metrics, es una clasificación integrada de las revistas de Ciencias sociales y Humanidades. Se pretende que los indicadores resultantes se utilicen en las agencias de evaluación nacionales. Divide a

10 https://web.archive.org/web/20150618035036/http://ec3.u gr.es/in-recj/

11 https://web.archive.org/web/20140713064650/http:/ec3.u gr.es/in-recs/

12 http://www.latindex.unam.mx/

$13 \mathrm{http}: / /$ miar.ub.edu/ las revistas en cinco categorías y puede consultarse en su página web y a través de Dialnet.

$D{ }^{16} E^{16}$ (no se actualiza desde 2013). Es la base de datos para la difusión y calidad editorial de las Revistas Españolas de Humanidades y Ciencias Sociales y Jurídicas. Es una colaboración entre CSIC y ANECA y elaborado por EPUC del CSIC. Proporciona diferentes criterios de calidad sobre la evaluación de las revistas, criterios Latindex y categoría de la revista en diferentes sistemas. La ANECA utiliza esta base de datos como referencia de calidad de las publicaciones españolas, en sus procesos de evaluación de profesorado.

\section{ENVÍO DEL ARTÍCULO}

Tras la selección de la revista se elige la sección en la que podría publicarse el artículo, consultando la información que al respecto proporciona la misma revista. Se recomienda observar la orientación y el número de autores de los artículos publicados en los últimos números y revisar los artículos publicados por la revista que pueden servir de orientación. Es importante tener en cuenta las normas de publicación de la revista y adaptarse a ellas. No respetar las normas puede suponer la devolución del artículo y una disminución de la credibilidad como autor.

Hay que elegir bien la primera opción de envío ya que habrá que esperar la respuesta de la editorial antes de intentarlo en otra revista. A tener en cuenta la existencia cada vez mayor de las denominadas revistas depredadoras, que sólo buscan ganar dinero aprovechándose de la necesidad de publicar que tienen los investigadores. Se trata de revistas que se cuentan por miles y que cobran cientos de dólares a los autores a cambio una publicación rápida y de una mediocre o inexistente revisión por pares. Aumenta así el número de revistas secuestradas, cuyo nombre copia el de una revistas de prestigio para confundir al científico ${ }^{17}$.

Por áreas temáticas, las webs de las bibliotecas universitarias ofrecen información sobre

14 http://epuc.cchs.csic.es/resh/

$15 \mathrm{http}: / / w w w . c l a s i f i c a c i o n c i r c . e s /$

16 http://dice.cindoc.csic.es/busqueda.php

17 http://francis.naukas.com/2016/01/26/la-explosion-depredadores-entre-las-revistas-cientificas/ 
dónde publicar por cada una de áreas temáticas. Por ejemplo pueden consultarse las guías y seminarios de la Biblioteca de la Universidad de Sevilla por áreas temáticas ${ }^{18}$.

\section{BIBLIOGRAFÍA}

1. Cardona-Grau D, Sorokin I, Leinwand G, Welliver $C$. Introducing the Twitter Impact Factor: An Objective Measure of Urology's Academic Impact on Twitter. Eur Urol Focus [Internet]. 2016 Apr [Citado el 13 de abril de 2016]. Disponible en: http://www.sciencedirect.com/science/arti cle/pii/S2405456916300086.

2. Roemer RC, Borchardt R. Meaningful metrics: A 21st century librarian's guide to bibliometrics, altmetrics, and research impact [Internet]. Chicago, Illinois: Association of College and Research Libraries; 2015. Disponible en: http://www.ala.org/acrl/sites/ala.org.acrl/fi les/content/publications/booksanddigitalr esources/digital/9780838987568_metrics_OA. pdf. 